\title{
A Participação da Mãe no Mercado de Trabalho e o Diferencial de Anos de Estudo por Gênero entre Adolescentes •
}

\author{
Ana Cecília de Almeida ${ }^{1}$ \\ João Eustáquio de Lima \\ Lorena Vieira Costa ${ }^{3}$
}

\section{Resumo}

Atualmente, o número de crianças que crescem em famílias cujas mães são ativas no mercado de trabalho é significativamente superior ao de gerações anteriores. Essa mudança demográfica envolve alterações quanto ao tempo e cuidado materno dedicado aos filhos, podendo afetar suas vidas atuais e prospecções futuras. Por isso, o objetivo desta pesquisa é contribuir para o debate acerca dos efeitos relacionados à participação da mãe no mercado de trabalho e a escolaridade dos filhos, medida pelas horas de trabalho. As estimações são obtidas por meio de variável instrumental como estratégica de identificação para isolar os efeitos causais entre a decisão pelo mercado de trabalho das mães e a escolaridade dos filhos, nas quais as condições do mercado de trabalho local são utilizadas como instrumentos. Entre os resultados, destaca-se que o aumento das horas trabalhadas da mãe reduz o nível educacional deles. Em relação ao diferencial educacional entre os sexos, observa-se que o efeito da mãe trabalhar não é responsável por introduzir diferenciais educacionais entre os sexos.

\section{Palavras-Chave}

Emprego Materno. Diferencial Educacional entre Gêneros.

- Artigo extraído da dissertação de mestrado "A participação da mãe no mercado de trabalho e o diferencial de anos de estudo por gênero entre adolescentes: evidência para o Brasil", apresentada à Universidade Federal de Viçosa, Viçosa, MG, Brasil.

1 Doutoranda - Departamento de Economia Rural - Universidade Federal de Viçosa (UFV)

Endereço: Avenida Purdue, s/n ${ }^{\circ}$ - Campus Universitário, Edifício Edson Potsch Magalhães, $1^{\circ}$ Andar, Sala 105 - CEP: 36570-900 - Viçosa/MG - Brasil - E-mail: ananinha6@hotmail.com https://orcid.org/0000-0001-8380-7202

2 Professor - Departamento de Economia Rural - Universidade Federal de Viçosa (UFV)

Endereço: Avenida Purdue, s/n $\mathrm{n}^{\circ}$ - Campus Universitário, Edifício Edson Potsch Magalhães, $1^{\circ}$ Andar, Sala 105 - CEP: 36570-900 -Viçosa/MG - Brasil - E-mail: jelima@ufv.br https://orcid.org/0000-0003-4404-4817

3 Professora - Departamento de Economia Rural - Universidade Federal de Viçosa (UFV) Endereço: Avenida Purdue, s/n ${ }^{\circ}$ - Campus Universitário, Edifício Edson Potsch Magalhães, $1^{\circ}$ Andar, Sala 105 - CEP: 36570-900 - Viçosa/MG - Brasil - E-mail: lorenavclelis@gmail.com https://orcid.org/0000-0002-0293-9842 Recebido: 09/05/2017. Aceite: 31/05/2018. 


\begin{abstract}
Currently, the number of children raised by working mothers is significantly higher than in previous generations. This demographic change involves shifts regarding to duration and type of childcare, which may affect children's lives. Accordingly, the objective of this research is to contribute to the debate about the effects regarding mother's labor force participation and the schooling of their children, measured by hours worked. The estimates are also performed using instrumental variables with identification strategy in order to isolate the causal effect between the decision by the mother's labor force and the schooling of their children, in which local labor market conditions are used as instruments. Among the results, it is noteworthy that the fact that the mother's worked hours decreases the children's education. Regarding the educational gender gap, mother's work is not responsible for introducing educational differentials between the sexes.
\end{abstract}

\title{
Keywords
}

Maternal Employment. Educational Differential between Sexes.

\section{Jel Classification}

121. J16. J22. J24.

\section{Introdução}

A questão sobre igualdade de gênero na educação faz parte das seis metas para melhorar os sistemas educacionais no mundo, assinadas por 164 países no ano 2000 no Fórum Mundial de Dakar. ${ }^{1}$ De acordo com os dados do Relatório de Monitoramento Global (UNESCO 2015), o progresso foi mais expressivo no ensino médio, nível em que $68 \%$ dos países conseguiram alcançar a paridade de gênero até 2015. Já no ensino fundamental, apenas $48 \%$ dos países tiveram sucesso até esse mesmo ano. A diferença entre o número de matrículas também reduziu, mesmo ainda permanecendo maior a probabilidade das meninas nunca se matricularem em relação aos meninos, $48 \%$ contra 37\%, respectivamente. Porém, quando matriculadas, elas apresentaram maiores chances de concluírem sua educação, pois apresentaram menor probabilidade de abandonar a escola (20\% contra $26 \%$ para os homens).

1 Segundo o Relatório de Monitoramento Global (UNESCO 2015), esse é o objetivo 5 do Compromisso de Educação para Todos (EPT): Paridade e igualdade de gênero, cuja meta era eliminar da educação primária e secundária as diferenças de gênero até 2005 e chegar à igualdade educacional entre os sexos, em todos os níveis educacionais, até 2015. O principal objetivo é garantir o acesso completo e equitativo das mulheres a uma educação básica de qualidade. 
No Brasil, não há paridade entre os sexos, e sim uma reversão do hiato em favor das mulheres, ou seja, as mulheres possuem em média mais anos de estudo do que os homens. Segundo Beltrão e Alves (2009), analisando os dados desagregados por coorte de idade, percebe-se que a reversão do gap educacional por gênero ocorreu primeiro entre as pessoas com idades entre 10 a 14 anos, na década de 1960. Essa mudança ocorreu inicialmente no ensino fundamental, evoluindo para o ensino médio, e, a partir de 1970, para o ensino superior.

Em uma análise para os anos mais recentes, observa-se na Figura 1 que as mulheres continuam estudando mais que os homens. As médias de escolaridade vêm crescendo para ambos os sexos, mas em todos os anos de análise as mulheres têm médias maiores que os homens.

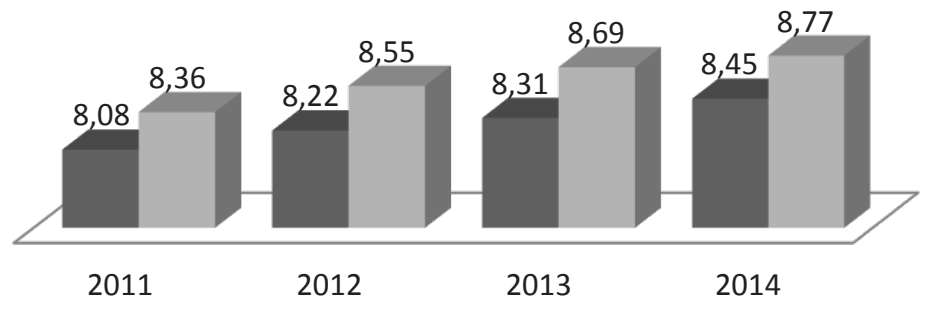

- Anos de estudo dos homens

Anos de estudo das mulheres

Figura 1- Média de anos de estudo da população brasileira por sexo

Fonte: Elaboração própria a partir de dados das PNADs de 2011 a 2014 (IBGE).

Uma das implicações da elevação da escolaridade das mulheres diz respeito à sua maior inserção no mercado de trabalho. No Brasil, verifica-se pelos dados da PNAD, representados na Figura 2 que entre os anos de 2004 a 2014, houve um aumento de 7,169 milhões de mulheres na população locada nos postos de trabalho. Em relação às mães, segundo Souza e RiosNeto (2008) nos grupos com idades entre 21 e 35 anos e com no mínimo dois filhos, a taxa de participação laboral passou de $11,3 \%$ no ano de 1970 , para 39,81\% em 2000. Além disso, 30\% da população feminina empregada em 2006 era representada por mulheres chefes do domicílio e, entre elas, $50,6 \%$ não eram casadas e moravam com seus filhos. 


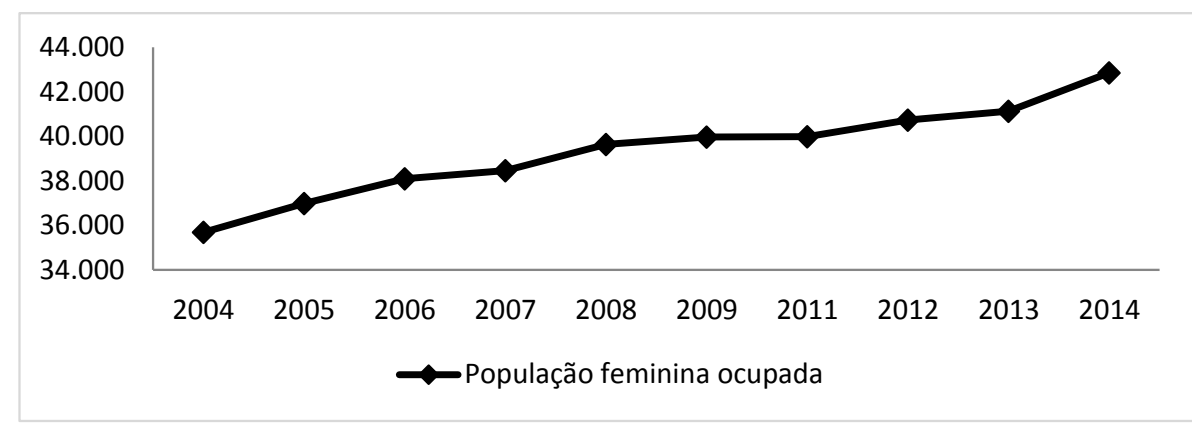

Figura 2- Quantidade de mulheres ocupadas maiores de 5 anos, em milhões - Brasil

Fonte: Elaboração própria a partir de dados das PNADs de 2004 a 2014 (IBGE) - com exceção dos dados de 2010, devido à sua ausência no ano censitário.

Essas estatísticas sugerem uma tendência de elevação da participação das mães no mercado de trabalho, um movimento importante no sentido de contribuir para a igualdade de oportunidades laborais entre os gêneros (ainda que persistam desigualdades salariais). Como resultado, tem-se observado uma redução no tempo da mãe em casa com os filhos, o que pode levar a diferentes implicações sobre os domicílios. Verificar o impacto dessas tendências é de suma importância dado que poderiam indicar efeitos benéficos do trabalho das mães sobre seus filhos (tanto por meio da elevação da renda quanto do empoderamento feminino e do exemplo a ser seguido) ou impactos prejudiciais (principalmente relacionados à redução do tempo de qualidade com os filhos associada a uma ausência de contrapartida dos cônjuges no cuidado com os adolescentes).

A literatura apresenta evidências de uma série de alterações comportamentais e demográficas dentro do domicílio, que podem ser resultado dessa maior participação das mulheres no mercado de trabalho. Um exemplo disso é o estudo de Ruhm (2008) para os Estado Unidos, que busca, por meio de três perspectivas, identificar o efeito de a mãe trabalhar sobre o desenvolvimento dos adolescentes: desenvolvimento cognitivo, problemas socioemocionais e saúde. Os resultados indicam que as horas de trabalho semanais das mães se associam a uma redução das notas dos filhos e de problemas comportamentais e a uma elevação na obesidade, no uso de cigarros e de bebidas alcoólicas. 
Nesse sentido, o objetivo do presente trabalho é verificar de que forma, no Brasil, a oferta de trabalho das mães relaciona-se com o diferencial no nível de escolaridade dos adolescentes de sexo diferentes, o que será analisado pelos anos de estudo. Assim, este trabalho tem a seguinte hipótese: a participação da mãe no mercado de trabalho, medida pelas horas por ela trabalhadas afeta de forma diferente os níveis de escolaridade dos filhos do sexo masculino e do sexo feminino, aumentando a diferença de anos de estudo entre os sexos. Embora a importância dessa relação seja consensualmente reconhecida na literatura internacional e pouco discutida na nacional, a magnitude e os sinais desse efeito são controversos principalmente devido à endogeneidade e a negligência quanto à correção desse problema (o que se buscou corrigir neste artigo).

Este trabalho, além de contribuir para o debate existente nos estudos que investigam a relação entre a vida laboral das mulheres e a educação dos filhos, traz resultados que permeiam a discussão quanto ao papel da mulher na sociedade brasileira. Assuntos tais como licenças parentais igualitárias, igualdade de oportunidades tanto em relação às condições no trabalho quanto no cuidado com filhos e empoderamento feminino são de suma importância para uma Nação que se pretende desenvolver. Segundo Duflo (2012), entende-se por empoderamento o melhoramento das possibilidades de acesso da mulher a fatores de desenvolvimento econômico, como acesso à saúde, educação, participação política e igualdades salariais. Para essa autora, o empoderamento da mulher pode acelerar o desenvolvimento econômico, ao mesmo tempo em que o desenvolvimento eleva a igualdade entre os sexos. A contínua discriminação - e ausência de oportunidades para elas - seria, assim, um entrave ao desenvolvimento.

\section{A Participação das Mães no Mercado de Trabalho e Efeitos Associa- dos sobre os Filhos}

Importantes efeitos domiciliares e econômicos resultam da maior participação das mães no mercado de trabalho e a reversão do hiato educacional de sexo em favor das mulheres pode ser um deles. Tais fatores têm sido amplamente estudados na literatura internacional e pouco avaliados no contexto nacional. Autores como González de San Román e De La Rica Goiricelaya (2012) e Fan, Fang e Markussen (2015) identificaram que 
filhos de sexos diferentes, com mães ativas no mercado de trabalho, tendem a ter diferenças educacionais, sendo o fato em média mais benéfico para as mulheres do que para os homens. Entretanto, outros autores divergem de opinião e consideram que não há diferença entre os sexos (Baum 2004 e Han; Waldfogel; Brooks-Gunn 2001).

Baum (2004) também sugere que a ausência da mãe quando empregada gera efeitos distintos dependendo da idade dos filhos, não sendo ainda claro na literatura em qual idade o efeito seria mais significativo. $\mathrm{O}$ desenvolvimento dos filhos tem na família a primeira fonte de influência. Ademais, segundo Anazawa et al. (2016), resultados futuros na vida do indivíduo em áreas como educação, saúde e mercado de trabalho podem ser explicados pelas condições iniciais da vida desse indivíduo. Essas condições agem através de fatores biológicos (pré-disposição genética da criança) e do ambiente social (condição socioeconômica e de saúde das famílias e estilo de interação entre os pais e filhos). Por isso, alguns trabalhos, como Hill e O'Neill (1994) defendem que o efeito da mãe trabalhar nos primeiros anos de vida da criança pode ser maior do que quando isso acontece em momentos posteriores da vida das crianças. Isso também decorre do fato de que na pré-adolescência e na adolescência outras pessoas passam a exercer maior influência na vida desses indivíduos, como professores e colegas.

Ainda de acordo com Baum (2004), o fato de a mãe trabalhar quando os filhos são adolescentes, tem um efeito maior na vida acadêmica de seus filhos, o que pode ser explicado não apenas pelo canal do modelo (role model channel), mas também pelo canal da renda. Pelo canal renda, o emprego materno aumenta os recursos financeiros da família, o que pode proporcionar aos filhos um melhor desempenho acadêmico, uma vez que poderão investir em recursos como aulas particulares, cursinhos e monitorias, além de poderem frequentar melhores escolas. Pelo canal do modelo (role model channel), ao tomar como exemplo suas mães, os filhos mais velhos conseguem ter maior consciência e discernimento sobre possíveis vantagens advindas do estudo.

Em uma análise empírica, Datcher-Loury (1988) avaliou o impacto do trabalho da genitora sobre a educação dos filhos (utilizando a média de anos de estudo, dados em painel e pessoas entre 20 e 26 anos). $\mathrm{O}$ autor encontrou resultados assimétricos entre os sexos: os filhos do sexo masculino têm seus anos de estudos reduzidos caso a mãe trabalhe quando pequenos, enquanto para as filhas, o efeito é oposto. Tanto Baydar e 
Brooks-Gunn (1991), quanto Brooks-Gunn, Han e Waldfogel (2002) e Fan, Fang e Markussen (2015), chegaram ao resultado de que a oferta de trabalho da mãe tem efeito assimétrico na educação dos filhos de sexos diferentes. Para as três pesquisas, o efeito é mais prejudicial para os filhos do sexo masculino. Baydar e Brooks-Gunn (1991), chegaram a essa conclusão analisando as notas das crianças de 3 a 4 anos, para os Estados Unidos. Enquanto Brooks-Gun, Han e Waldfogel (2002), analisaram as notas de crianças de 1 a 3 anos de idade.

Fan, Fang e Markussen (2015) analisam para dois países diferentes: em dados em painel para Noruega e em seção cruzada para os Estados Unidos. Para o primeiro, foram utilizados os dados de pessoas com até dezessete anos. Concluíram que, após controlar a endogeneidade, o fato de a mãe estar no mercado de trabalho aumenta a probabilidade das mulheres terem ensino superior e para os homens o efeito é o inverso, isso para todos os grupos de idade (considerando o fato de a mãe trabalhar quando os filhos tinham 0 a 5 anos de idade, 6 a 11, e 12 a 17). Para os Estados Unidos, o resultado estimado foi que a oferta de trabalho da mãe casada está relacionada positivamente com o gap educacional (diferença entre a conclusão do ensino superior para mulheres e para homens).

$\mathrm{Na}$ literatura nacional, Vieira et al. (2016) analisam a relação entre a renda e a oferta de trabalho dos pais nas decisões entre trabalho e estudo dos adolescentes. Esses autores encontraram que tanto a participação da mãe quanto a do pai no mercado de trabalho diminuem a probabilidade dos adolescentes de apenas estudarem. Dessa forma, os adolescentes cujos pais estão trabalhando tendem a dividir o seu tempo não somente com os estudos, mas com outras atividades, como cuidar dos irmãos e da casa. Além disso, o trabalho também aponta que o impacto do trabalho da mãe é maior que o do pai, apenas na decisão dos filhos só trabalharem em relação a ficarem na situação "nem-nem". A renda de ambos os pais apenas diminui a probabilidade dos filhos escolherem a opção de apenas trabalhar, pois com maior renda familiar haverá maior possibilidade de investir na educação dos filhos podendo haver uma maior dedicação destes aos estudos, por não ter tanta necessidade de complementar a renda da família.

Um dos meios utilizados por Aquino e Pazello (2011) para verificar o papel da família sobre o desempenho escolar das crianças brasileiras foi a análise entre o trabalho materno e a probabilidade de aprovação dos seus filhos. Foram utilizados dois períodos diferentes, de 1986 a 1995 e de 2002 a 
2006. Para ambos os períodos, o impacto da entrada da mãe no mercado de trabalho na aprovação dos filhos foi negativo. Para a amostra mais antiga esse impacto é menor do que para as gerações mais novas. Quando analisado o trabalho da mãe em diferentes jornadas de trabalho, o fato de a mãe trabalhar meio período em relação a não trabalhar aumentou a probabilidade de aprovação das crianças, mas esse resultado não foi estatisticamente significativo.

Arouck (2015) também analisa esta relação no Brasil, restringindo seu estudo à população do Rio Grande do Sul. Os resultados obtidos seguem similares: a participação da mãe na PEA (População Economicamente Ativa) diminui a probabilidade de aprovação escolar do filho, mas esse efeito reduz com o aumento da renda da família e torna-se não significativo. Além disso, também conclui que o efeito do trabalho da mãe é passageiro. Não obstante, não somente as características de trabalho dos pais são responsáveis por explicar a educação dos filhos, outros fatores também são importantes, como a renda, a composição familiar, a escolaridade e as condições ou práticas de saúde dos pais e condições de saúde dos recémnascidos (Anazawa et al. 2016).

\section{Marco Teórico: A Oferta de Trabalho das Mães}

Busca-se na sociologia e na economia teorias para prever os efeitos do trabalho materno sobre as crianças e adolescentes. Na literatura econômica, as crianças e adolescentes são percebidos como um bem na produção da família. Ao nascerem, possuem habilidades inatas que devem ser desenvolvidas durante seus anos de vida. Para isso, é necessária a presença dos pais, dos irmãos, professores e outras pessoas que serão importantes para seu desenvolvimento, como também uma escola de qualidade, livros, brinquedos e artigos esportivos. Para obtenção destes, faz-se necessário uma boa renda dos familiares, que pode ser adquirida trocando tempo com os filhos por participação no mercado de trabalho. Às famílias, cabem tomar suas decisões levando em conta a alocação de seu tempo, o consumo e as decisões de investimentos para o desenvolvimento de seus filhos. 
De acordo com Baum (2004), na sociologia, a mais comumente utilizada é a "teoria do modelo" ou canal do modelo (role model channel). Essa teoria sugere que os pais são como um exemplo a ser seguido pelos seus filhos, ou seja, pais de sucesso implicitamente impulsionam seus filhos a também o terem. Os pais tomam a decisão de trabalhar ou não e, decidem também, sobre qual a melhor quantidade de horas trabalhadas, tendo como principal objetivo o aprimoramento das habilidades das crianças e adolescentes. Mas deve-se ter em mente também que, essa ausência da mãe em casa pode afetar de maneiras diferentes os filhos de sexos opostos.

Segundo Fan, Fang e Markussen (2015), a participação da mãe no mercado de trabalho pode afetar de maneira distinta os filhos de diferentes sexos e isso pode ocorrer devido a três canais: o canal da renda, do modelo e da produção. Em relação ao canal da renda, esses pesquisadores não encontraram impacto significativo desse efeito no diferencial educacional de sexo. Pelo canal do modelo (role model channel), os filhos veem pelo exemplo da mãe os retornos que a educação traz e, dessa forma, são mais conscientizados e buscam estudar e se dedicar mais, sendo esse resultado maior para os filhos do sexo feminino. ${ }^{2}$ Já no canal da produção (production channel), a produtividade marginal do tempo gasto com os filhos pode gerar um efeito positivo maior na educação dos meninos do que das meninas. ${ }^{3}$ Assim, ter menos tempo com os filhos devido ao trabalho fora de casa prejudica mais a educação dos filhos do sexo masculino. Esses dois efeitos juntos tendem a introduzir um gap de gênero na educação dos filhos em favor das mulheres.

Todavia, esses dois canais, de acordo com Fan, Fang e Markussen (2015), são difíceis de serem identificados separadamente, mas é possível reconhecer em qual momento na vida dos filhos cada canal contribui mais ou menos. Esses pesquisadores defendem que existem dois meios pelos quais o canal do modelo (role model channel) age: de maneira consciente e inconsciente. Nos primeiros anos de vida das crianças o efeito inconsciente é maior, mas vai diminuindo com a idade. O contrário acontece com o efeito

2 As meninas seguirem mais o exemplo dos pais que os meninos pode ocorrer devido a algumas características comportamentais que diferem entre esses os sexos, expostas por Muller (1998): os meninos são mais propensos a se envolverem com outras pessoas sem ser da família e essas pessoas podem influenciá-los. Ao contrário, as meninas são mais inclinadas a construir relacionamentos de apoio com os pais e tendem a conversar mais e a se entenderem melhor.

3 Uma possível explicação para isso está no fato de que os meninos são diagnosticados com Transtorno de Déficit de Atenção / Hiperatividade (TDAH) com maior frequência do que as meninas. Segundo Cardoso, Sabbag, Beltrame (2007), essa doença afeta a capacidade da criança em controlar seu tempo e compromete a sua vontade, o que prejudica a sua concentração e seu aprendizado. Por isso, essas crianças precisam de mais atenção e cuidado para conseguirem aprender, sendo então o tempo da mãe, com os cuidados ativos, necessário. 
consciente do canal do modelo (role model channel). Assim, quando mais velhos, os filhos já conseguem perceber pelo exemplo da mãe trabalhando, que estudar é um meio de conseguir entrar no mercado de trabalho e ter sucesso profissional. A outra parte é explicada pelo canal da produção (production channel) que, como mencionado anteriormente, é mais produtivo para os meninos do que para as meninas, mas essa diferença diminui com a idade das crianças segundo o estudo de Fan, Fang e Markussen (2015). Dessa forma, como a presente pesquisa analisa os adolescentes, é de se esperar que o canal do modelo (role model channel) sobreponha o canal da produção (production channel), mas os dois juntos podem explicar o aumento no diferencial educacional de gênero devido à presença da mãe no mercado de trabalho.

\section{Metodologia}

\section{1. O efeito da Oferta de Trabalho da Mãe sobre o Diferencial Educacional entre os Sexos dos Adolescentes}

Para identificar o efeito pretendido neste artigo, foram utilizados os dados da Pesquisa Nacional por Amostra de Domicílios (PNAD), referente aos anos de 2004 a 2014. Os dados de cada período da pesquisa foram empilhados de forma a obter variações no tempo e nas unidades de seção cruzada. A equação de interesse é especificada da seguinte forma:

$$
\begin{gathered}
y_{i, s, t}=\rho+\operatorname{shtrab}_{m \tilde{a} e} e_{i, s, t}+\alpha\left(\text { htrab }_{\text {mãe }} i, s, t\right. \\
\left.+\operatorname{sexo}_{\text {filho }_{i, s, t}}\right)+\operatorname{sexo}_{\text {filho }_{i, s, t}}+X_{i, s, t}^{\prime} \sigma \\
+V_{i, s, t}^{\prime} \beta+\phi \text { DummyAno }_{t}+\psi \text { DummyLocal }_{s}+e_{i, s}
\end{gathered}
$$

em quese $y_{i, s, t}$ refere à média de anos de estudo do adolescente $i$, residente na Região Metropolitana $s$ no período da pesquisa $t$, sendo que cada série concluída com aprovação corresponde a um ano a mais de estudo.

Sobre essa variável, cabe destacar que na literatura internacional a maioria dos trabalhos sobre o tema utiliza as notas alcançadas como Proxy para educação, tanto nos primeiros anos de vida referentes às habilidades cognitivas (Blau; Grossberg 1990), quanto na relação com as notas na escola (Baum 2004 e Ruhm 2008). Alguns autores analisam a probabilidade de 
entrar no ensino superior (Fan; Fang; Markussen 2015) e poucos analisam anos de estudo (Datcher-Loury 1988). Devido às limitações das bases de dados brasileiras, a forma mais factível de considerar a educação dos adolescentes foi por meio dos seus anos de estudo.

A variável de interesse é $h$ trab $b_{m a ̃ e}{ }_{i, s, t}$, que se refere às horas trabalhadas por semana pela mãe, variando de 0 a 98 horas. Tem-se a variável sexo $_{\text {filho }_{i, s, t^{\prime}}}$ que recebe valor de um (1) se o filho $i$ é do sexo masculino, e zero (0) caso contrário. Entre as variáveis independentes tem-se um vetor de variáveis de controle $\left(X_{i, s, t}^{\prime}\right)$ que contém as seguintes variáveis: raça, educação da mãe, educação do pai, idade da mãe no nascimento do filho, estado civil da mãe, tamanho da família, renda familiar mensal e o trabalho do filho.

No vetor $V_{i, s, t}^{\prime}$, foram inseridas dummies de coorte para os filhos e para as mães, a fim de controlar diferenças entre indivíduos que nasceram em períodos distintos. Além disso, é adicionado ao modelo o efeito fixo de região metropolitana (dummies de localidade) que foi utilizado para capturar tudo o que é comum aos indivíduos desta localidade e o efeito fixo de ano (dummies de ano), objetivando capturar condições que se alteram em cada período de tempo.

Assim como feito por Fan, Fang e Markussen (2015), Baum (2004) e Blau e Grossberg (1990), neste artigo o efeito do trabalho da mãe sobre a escolaridade dos filhos de diferentes sexos é captado pela interação entre horas trabalhadas da mãe e o sexo do filho $\left(\right.$ htrab $_{m a \tilde{e} i, s, t} * \operatorname{sexo}_{\text {filho }_{i, s, t^{\prime}}}$, como descrito na Equação (1). ${ }^{4}$ Nesse caso, o efeito marginal do trabalho da mãe sobre os anos de estudo dos meninos em relação às meninas é dado pelo coeficiente $\alpha^{5}$. Caso ele seja negativo e estatisticamente significativo, então o aumento de uma hora de trabalho da mãe é mais prejudicial (ou menos benéfico) para os meninos do que para as meninas, aumentando o diferencial de anos de estudo entre os sexos.

${ }^{4}$ As variáveis de controle: estado civil da mãe, renda familiar e anos de estudo dos pais também estão interagidas com a dummy de sexo do filho, buscando controlar as diferenças que essas variáveis estabelecem entre os filhos de diferentes sexos.

5 Dessa forma, $\varsigma$ é o efeito marginal de a mãe trabalhar sobre a média de anos de estudo das meninas e $\varsigma+\alpha$ é o efeito marginal sobre os meninos. 


\subsection{Estratégia de Identificação: O Uso das Condições Locais do Mercado de Trabalho como Instrumento para as Horas de Trabalho das Mães}

A estratégia mais simples para identificar a relação entre a oferta de trabalho da mãe e o diferencial educacional de gênero dos filhos seria por meio das estimativas de Mínimos Quadrados Ordinários (MQO). Uma simples regressão linear, no entanto, deteria a suposição de que a oferta de trabalho da mãe - a principal variável explicativa de interesse deste estudo- seja uma variável independente e que não tenha correlação com o termo do erro da equação que explica o nível de escolaridade de filhos e filhas. Não obstante, essa suposição oferece sérias limitações quanto à interpretação causal do efeito de interesse. Mudanças nas características não observadas das mães, associadas à qualidade dos cuidados com os filhos, podem ser correlacionadas com o emprego materno, o que faria com que existisse uma possível fonte de viés de endogeneidade (James-Burdumy 2005).

Conforme Blau e Grossberg (1990), a decisão de trabalhar é uma autosseleção, e essa decisão pode ser tomada, em algumas famílias, por meio da comparação entre a produtividade da mãe em casa e aquela obtida no mercado de trabalho. Tais produtividades se relacionam com características não observadas, por exemplo, dependem das habilidades da mãe no mercado de trabalho e nos cuidados com os filhos. Assim, mães que trabalham podem ter características não observáveis que se relacionam com a obtenção de maiores (empreendedorismo, dinamismo, habilidades) ou menores (cansaço, ausência de compartilhamento de tarefas domésticas entre cônjuges) níveis de escolaridade de seus filhos. Na ausência do controle de tais características, os resultados expressos pelo MQO não refletiriam efeitos causais da decisão de emprego da mãe, mas refletiriam em parte efeitos de tais variáveis não incluídas no modelo.

A estratégia empírica adotada neste trabalho oferece um meio de contornar este problema. Por meio da abordagem de variáveis instrumentais, buscouse por uma variação exógena sobre a decisão de emprego das mães brasileiras (mais precisamente, a quantidade de horas trabalhadas). O intuito é retirar da decisão de trabalho das mulheres os aspectos não observados que o determinam (como empreendedorismo, habilidade e outros).

O instrumento empregado neste trabalho é o mesmo utilizado por Anderson, Butcher e Levine (2003), Baum (2003) e James-Burdumy (2005): condição do mercado de trabalho local. Visto que os municípios não podem ser identificados a partir da base de dados utilizada neste es- 
tudo (PNAD), a princípio, duas opções seriam possíveis quanto à variável instrumental relacionada às condições de trabalho locais: essas poderiam ser obtidas por meio de dados estaduais ou das regiões metropolitanas brasileiras. As condições do mercado de trabalho no estado onde se encontram as mulheres pesquisadas é um mecanismo para a decisão de emprego dessas. Essas condições tornam-se um instrumento que esbarra no problema relacionado à heterogeneidade dentro das unidades da federação. Ao se agregarem os dados referentes ao mercado de trabalho ao nível estadual, a variável instrumental poderia estar comprometida pelo baixo poder de explicação das decisões das mães. ${ }^{6}$

Para lidar com essa questão, optou-se por delimitar a amostra às regiões metropolitanas. ${ }^{7}$ As condições agregadas do mercado de trabalho dessas regiões seriam uma fonte de variação exógena (e potencialmente forte) da decisão de trabalho das mães (com idade entre 15 e 49 anos) moradoras dessas regiões. As incertezas e precariedades dos mercados de trabalho locais são apontadas por Leone (2000) como importantes determinantes da decisão de trabalho dos membros da família (inclusive das mulheres).

James-Burdumy (2005) ainda aponta que tais instrumentos - relacionados ao mercado de trabalho - são variáveis externas às mães, ou seja, não são características maternas, e por isso, não há razões para que elas não sejam ortogonais ao termo do erro da equação que identifica seu efeito sobre a educação dos filhos. Assim, esta estratégia baseia-se na hipótese de que a única forma pela qual as variáveis relacionadas às condições agregadas do mercado de trabalho das regiões metropolitanas afetam os níveis de escolaridade das crianças e adolescentes sejam por meio da decisão de emprego da mãe - depois de controlados outros fatores observáveis que também determinam o fenômeno em estudo.

Dentre as variáveis que refletem o mercado de trabalho agregado, foram testados quatorze potenciais instrumentos ${ }^{8}$ propostos por Baum (2003) para a variável endógena deste trabalho (horas de trabalho da mãe). A fim

6 Instrumentos fracos elevam a imprecisão das estimativas dos erros padrões e podem acentuar o viés de amostras finitas. Isto é, o estimador de variáveis instrumentais é viesado em pequenas amostras, ainda que consistente assintoticamente. Entretanto, o uso de instrumentos fracos acentua tal viés, mesmo em amostras com grande número de observações (Cameron; Trivedi 2005).

7 As regiões metropolitanas utilizadas foram as especificadas pela PNAD: regiões metropolitanas do Pará, Ceará, Pernambuco, Bahia, Minas Gerais, Rio de Janeiro, São Paulo, Paraná, Rio Grande do Sul e Distrito Federal.

8 Esses instrumentos foram testados quanto a sua validade pelo teste de sobreidentificação, cuja hipótese nula supõe ausência de correlação entre os instrumentos e o termo do erro e no tocante à sua relevância, por meio do teste de significância conjunta dos instrumentos no primeiro estágio da regressão. 
de evitar um número grande de instrumentos, que eleva a possibilidade de instrumentos fracos, além das devidas considerações a respeito da relevância e validade dos mesmos, definiram-se, afinal, duas variáveis instrumentais: população empregada no setor de comércio e no setor industrial. Com o intuito de identificar os efeitos diferenciais entre filhos de sexos diferentes, essas variáveis, assim como suas interações com a variável referente ao sexo do filho, foram utilizadas como instrumentos para a variável de interação entre o emprego materno e dummies indicativas do sexo do adolescente (conforme sugere Wooldridge (2002).

\subsection{Fonte e Tratamento dos Dados}

Os dados utilizados nesse artigo, como já mencionado anteriormente, são retirados da Pesquisa Nacional por Amostra de Domicílios (PNAD), disponibilizados pelo Instituto Brasileiro de Geografia e Estatística (IBGE) nos anos de 2004 a 2014. São consideradas na amostra apenas as pessoas que residem nas Regiões Metropolitanas. Os instrumentos também foram retirados das PNADs de 2004 a 2014, sendo estes agregados por Região Metropolitana, por meio do Sistema IBGE de Recuperação Automática (SIDRA).

A PNAD é uma pesquisa realizada anualmente e contém informações tanto do indivíduo, quanto do seu domicílio (neste trabalho serão utilizadas, informações do indivíduo) e tem como finalidade fornecer informações para auxiliar em estudos de desenvolvimento socioeconômico do Brasil. Algumas de suas informações são de periodicidade variável, como migração, fecundidade e saúde. Além disso, a PNAD é uma pesquisa amostral "complexa", pois incorpora níveis de complexidade como: estratificação das unidades de amostragem, probabilidades desiguais de seleção em um ou mais estágio, conglomeração (seleção de amostras em diferentes estágios) e ajustes de pesos amostrais. Isso implica que todas as análises estatísticas e econométricas devem incluir as variáveis amostrais, sendo essas as variáveis que definem o tamanho da amostra, representadas de modo geral, pelos estratos e pesos amostrais. 
A base de dados está em nível de indivíduos e é limitada aos adolescentes entre 12 a 18 anos de idade e que moram com a mãe. ${ }^{9}$ Essa restrição é importante, pois o objetivo deste estudo é mensurar se a ausência da mãe em casa, devido à sua participação no mercado de trabalho, afeta a educação de seus filhos, e se esse efeito é assimétrico entre os adolescentes de sexo diferentes. Além disso, a amostra foi limitada às mães que tinham entre 15 e 49 anos de idade. No total, a amostra é formada por 109.849 adolescentes, sendo 56.652 meninos e 53.197 meninas.

\section{Resultados}

\subsection{Perfil dos Adolescentes e de suas Famílias por Sexo}

Nesta subseção são analisadas as características médias dos adolescentes e de suas famílias. ${ }^{10}$ A Figura 3 mostra a média de anos de estudo para os adolescentes de diferentes sexos para o período de análise. Observa-se que em todos os anos os homens estudam menos que as mulheres e essa diferença é estatisticamente significativa. A partir de 2011, há uma pequena diminuição do gap educacional de gênero, e pode-se perceber que a média de anos de estudo tanto das meninas quanto dos meninos aumentou de 2004 a 2014 em 0,3 anos.

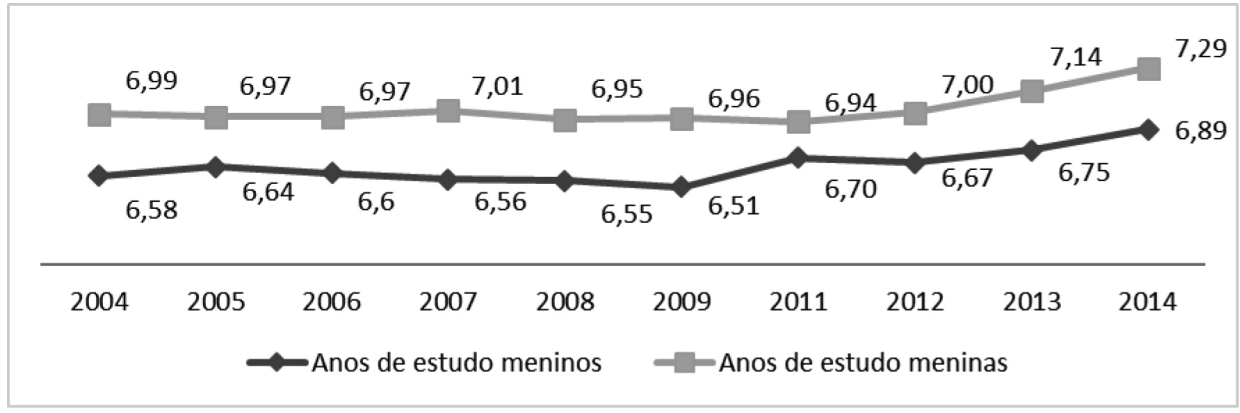

Figura 3- Média de anos de estudo dos adolescentes das regiões metropolitanas por sexo Fonte: Elaboração própria a partir de dados das PNADs de 2004 a 2014 (IBGE), com exceção da de 2010 pela sua ausência no ano censitário.

9 São consideradas todas as mães na amostra, podendo ter um ou mais filhos.

${ }^{10}$ Para o cálculo de todas as médias é utilizado o fator de expansão amostral. Além disso, é controlado o efeito de composição dentro da própria família, por meio da seleção aleatória de um integrante da família para compor as estatísticas descritivas da mãe, multiplicadas pelo número de filhos. 
As próximas análises são referentes às características das famílias dos adolescentes, sendo essas divididas em três grupos. O primeiro grupo corresponde a toda a amostra, não fazendo distinção entre os sexos. Já no segundo e terceiro grupos a amostra foi dividida entre adolescentes de sexos diferentes. Assim, no segundo grupo tem que ter pelo menos uma menina na família e no terceiro pelo menos um menino, mas uma mesma família pode ter filhos do sexo masculino e feminino.

Em relação às características de trabalho das mães, observa-se pelas Figuras 4 e 5 que tanto a participação delas no mercado de trabalho quanto as horas por elas trabalhadas aumentaram nesses 10 anos. Em média, 65\% das mães estavam trabalhando nesse período, sendo a média de horas por elas trabalhadas em torno de 24 horas semanais. Pode-se perceber também que entre os anos de 2009 a 2013, as mães de meninas trabalharam mais, mas não há um padrão em todos os anos da análise.

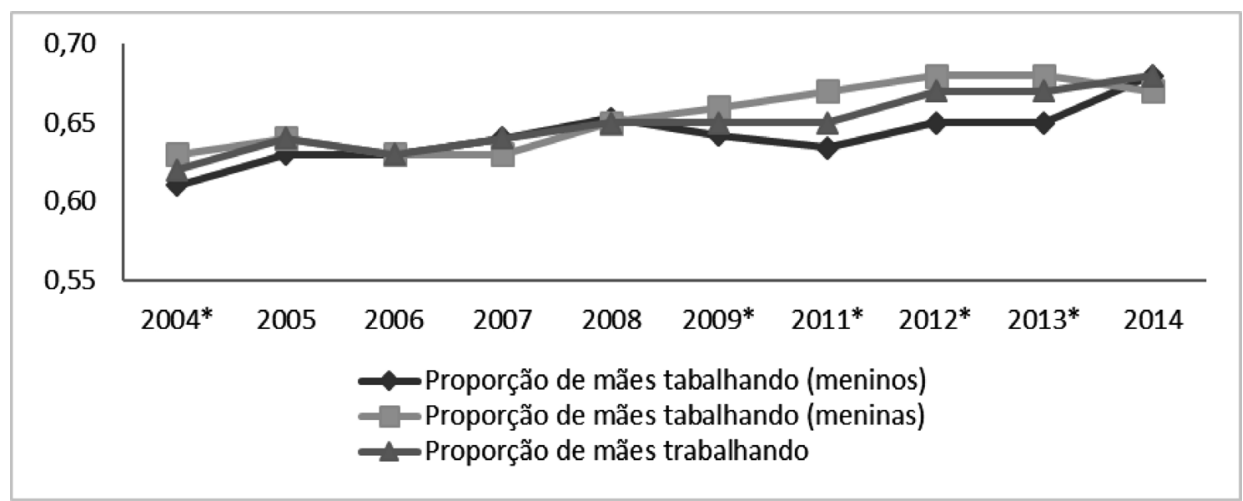

Figura 4 Proporção de mães trabalhando nas regiões metropolitanas

Fonte: Elaboração própria a partir de dados das PNADs de 2004 a 2014 (IBGE), com exceção da de 2010 pela sua ausência no ano censitário.

Nota: $\left({ }^{*}\right)$ significa que a diferença entre sexo naquele ano foi estatisticamente significativa. 


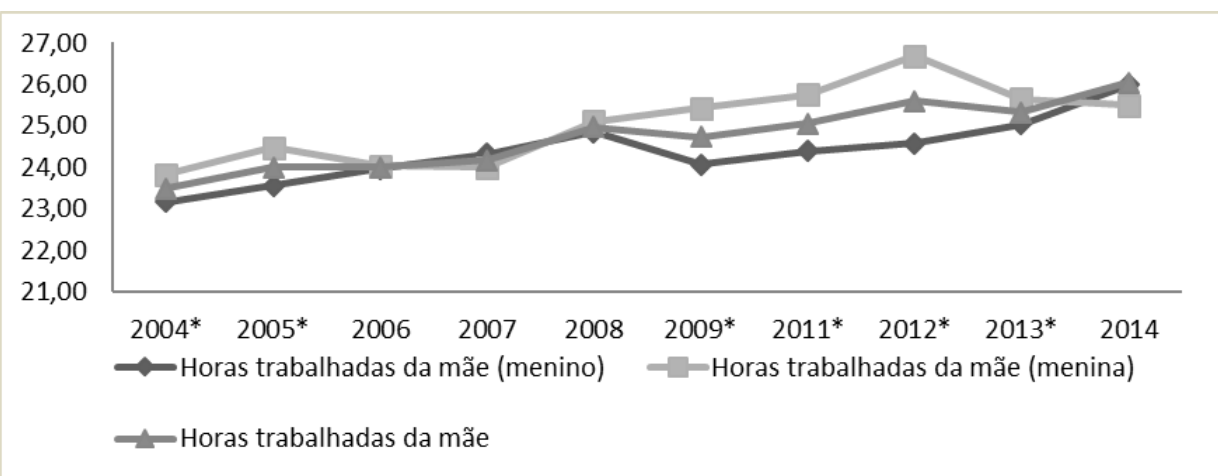

\section{Figura 5 - Horas trabalhadas das mães residentes nas regiões metropolitanas}

Fonte: Elaboração própria a partir de dados das PNADs de 2004 a 2014 (IBGE), com exceção da de 2010 pela sua ausência no ano censitário.

Nota: $\left({ }^{*}\right)$ significa que a diferença entre sexo naquele ano foi estatisticamente significativa.

De acordo com Aguas (2009), o sexo e a idade dos filhos são variáveis importantes para explicar a participação da mãe no mercado de traba1ho. A presença de meninas adolescentes, com idade superior a 10 anos, está relacionada a uma maior oferta de trabalho da mãe, o que pode ser explicado pelo fato de que essas adolescentes muitas vezes assumem as responsabilidades maternas no domicílio, tanto no cuidado da casa quanto no cuidado com os irmãos.

As características da família (background familiar) são também importantes para explicar a escolaridade dos adolescentes. Primeiro, é importante ver o perfil dos adolescentes em relação à educação dos seus pais. Por meio da Tabela 1, verifica-se que na amostra total as mães têm educação um pouco mais elevada do que os pais, sendo que aquelas possuem em média 8,33 anos de estudo, enquanto estes $8,16 .{ }^{11} \mathrm{O}$ mesmo acontece quando a amostra é dividida por sexo dos filhos. Não há diferença significativa entre o nível de educação das mães e dos pais para diferentes sexos dos filhos.

Conforme descrito na Tabela 1, em média, a renda familiar dos adolescentes de toda a amostra é de $\mathrm{R} \$ 2.391,93$ reais por mês, mas não há diferença de renda familiar para famílias com pelo menos um menino ou uma menina. Já em relação à composição da família, observa-se que $23 \%$ da amostra

11 Foram feitos, nessa subseção, todos os testes de médias entre os dois sexos. O resultado encontra-se mencionado no texto. 
é formada por famílias monoparentais (mãe sem cônjuge). A maior porcentagem de mães solteiras é para as mães de meninas (24\% contra $22 \%$ ).

Tabela 1 - Perfil dos adolescentes quanto ao background familiar, para toda amostra e por sexo - 2004 a 2014

\begin{tabular}{|c|c|c|c|c|c|c|}
\hline \multirow{3}{*}{ Todas as Mães } & & & \multicolumn{4}{|c|}{ Filhos } \\
\hline & & & \multicolumn{2}{|c|}{ Meninas } & \multicolumn{2}{|c|}{ Meninos } \\
\hline & Média & Desvio-padrão & Média & Desvio-padrão & Média & Desvio-padrão \\
\hline Anos estudo da mãe & 8,33 & 3,99 & 8.35 & 3,99 & 8,32 & 3,99 \\
\hline Anos estudo do pai & 8,16 & 4,10 & 8,18 & 4,10 & 8,15 & 4,11 \\
\hline Renda familiar mensal & $2.391,9$ & $3.387,7$ & $2.380,5$ & $3.429,6$ & $2.402,7$ & $3.347,5$ \\
\hline Mãe solteira (proporção) & 0,23 & 0,42 & 0,24 & 0,42 & 0,22 & 0,42 \\
\hline Tamanho da família & 2,28 & 1,09 & 2,27 & 1,10 & 2,29 & 1,08 \\
\hline Idade da mãe & 39,61 & 5,32 & 39.62 & 5,33 & 39.60 & 5,32 \\
\hline $\begin{array}{l}\text { Proporção de adolescentes } \\
\text { trabalhando }\end{array}$ & 0,29 & 0,45 & 0,28 & 0,45 & 0,31 & 0,46 \\
\hline Número de observações & \multicolumn{2}{|r|}{109.849} & \multicolumn{2}{|r|}{56.652} & \multicolumn{2}{|c|}{53.197} \\
\hline
\end{tabular}

Fonte: Elaboração própria com dados da pesquisa.

Com apenas 2,28 filhos em média, as famílias são pequenas. A idade média da mãe é de 39,61 anos e não foi encontrada diferença significativa entre as médias dessas duas últimas variáveis em relação aos filhos serem de sexos diferentes. Pode-se observar também que 29\% dos adolescentes estão exercendo alguma atividade que aufere renda para a família, sendo que os meninos estão mais presentes no mercado de trabalho do que as meninas ( $31 \%$ contra $28 \%)$.

A partir dos dados fornecidos pela amostra, esta subseção possibilitou a descrição do perfil dos adolescentes de diferentes sexos em relação às suas próprias características, as de suas famílias e a do mercado de trabalho da mãe. As diferenças entre as médias das estatísticas descritivas de cada variável para os meninos e para as meninas foram muito pequenas e seguiram as médias das variáveis da amostra total. 


\subsection{Efeito do Trabalho da Mãe sobre os Anos de Estudo dos Filhos de Dife- rentes Sexos}

Nesta subseção, apresentam-se os resultados das estimações da Equação (1), que tem o objetivo de identificar o efeito da intensidade laboral da mãe, por meio das horas trabalhadas por semana, sobre a escolaridade dos filhos e sobre o diferencial educacional de gênero.

Os instrumentos válidos foram: população empregada no setor de comércio e no setor industrial por Região Metropolitana e suas interações com a variável binária para sexo do filho. Pode-se concluir, por meio do teste F calculado, que os instrumentos para verificação dos efeitos da mãe no mercado de trabalho, nos filhos de ambos os sexos, são fracos, enquanto que ao analisar o efeito sobre os filhos de sexos diferentes os instrumentos foram fortes. Contudo, pelos resultados referentes ao primeiro estágio das estimações, observa-se que os instrumentos foram significativos para explicar tanto as horas trabalhadas da mãe quanto a interação dessa variável com o sexo dos filhos. A relação encontrada entre os instrumentos (população empregada no setor industrial e comercial) e as horas de trabalho da mãe é positiva. ${ }^{12}$

$\mathrm{Na}$ Tabela 2, encontram-se os resultados referentes às estimações da Equação (1) por meio do estimador de Mínimos Quadrados Ordinários (MQO) e estimador de variáveis instrumentais por meio do Método dos Momentos Generalizados (GMM). Além de permitir comparação das estimativas, testa-se a adequação dos estimadores por meio do teste DurbinWu-Hausman (DWH). O resultado desse teste confirma a adequação da estimação via variáveis instrumentais. Além disso, nesta pesquisa, para cada método, apresentam-se duas especificações alternativas: a primeira fornece efeitos médios sobre os adolescentes brasileiros e a segunda considera efeitos diferenciais de acordo com os sexos dos filhos. Inicialmente analisando as especificações de número (1), nota-se que o coeficiente referente ao trabalho materno é positivo e não significativo quando a equação é estimada via MQO. Isso significa que o viés do MQO (devido à endogeneidade quanto à decisão de oferta de trabalho das mães) age no sentido de evidenciar uma relação positiva e não significativa entre as horas trabalhadas pelas mães e o nível médio de escolaridade dos filhos.

12 Os resultados da estimação de primeiro estágio podem ser obtidos diretamente com os autores. 
Quando essa fonte de endogeneidade é tratada no contexto das variáveis instrumentais, tem-se um efeito negativo e estatisticamente significativo. Observa-se então, que quanto maior a oferta de trabalho da genitora, menor é o nível de capital humano do filho (especificação (1), via VI). Uma hora a mais trabalhada pela mãe reduz, em média, 0,095 os anos de estudo dos filhos, tudo o mais constante. Na mesma linha, Baum (2003), James-Burdumy (2005), Aquino e Pazello (2011) e Arouck (2015) também encontram efeitos negativos do trabalho da mãe sobre o desenvolvimento cognitivo dos filhos. Segundo James-Burdumy (2005), esse resultado pode acontecer devido ao maior estresse e cansaço causado pelo trabalho.

Verifica-se também pelos resultados da especificação (1) via VI, expressos na Tabela 2, que a maior escolaridade dos pais se associa positivamente ao número médio de anos de escolaridade dos adolescentes. O efeito marginal da escolaridade da mãe sobre os anos de estudo dos adolescentes é de 0,154 , enquanto que o do pai é 0,029 , tudo mais constante. Além disso, o fato dos filhos serem brancos em relação às outras raças também está associado a uma melhora em seus níveis de escolaridade, sendo esse efeito de 0,002 . 
Tabela 2- Efeito das horas trabalhadas pelas mães sobre os anos de estudo dos adolescentes e sobre o diferencial educacional de gênero, MQO e VI- 2004 a 2014

\begin{tabular}{|c|c|c|c|c|}
\hline & \multicolumn{2}{|c|}{ MQO } & \multicolumn{2}{|c|}{ VI } \\
\hline & (1) & (2) & (1) & (2) \\
\hline Horas de trabalho da mãe & $\begin{array}{l}0,0001 \\
(0,000)\end{array}$ & $\begin{array}{c}0,001 \\
(0,000)\end{array}$ & $\begin{array}{c}-0,095 * * \\
(0,044)\end{array}$ & $\begin{array}{c}-0,160 * * * \\
(0,053)\end{array}$ \\
\hline Horas trabalho da mãe* masculino & - & $\begin{array}{c}-0,001 * * \\
(0,001)\end{array}$ & - & $\begin{array}{c}0,004 \\
(0,019)\end{array}$ \\
\hline Casada & $\begin{array}{l}-0,219 \\
(0,154)\end{array}$ & $\begin{array}{l}-0,147 \\
(0,236)\end{array}$ & $\begin{array}{c}-1,527 * * \\
(0,644)\end{array}$ & $\begin{array}{c}-2,585^{* * *} \\
(0,913)\end{array}$ \\
\hline Casada*Masculino & - & $\begin{array}{c}0,048 \\
(0,111)\end{array}$ & & $\begin{array}{l}-0,125 \\
(0,219)\end{array}$ \\
\hline Renda familiar & $\begin{array}{c}0,012 * * * \\
(0,002)\end{array}$ & $\begin{array}{c}0,010^{* * *} \\
(0,002)\end{array}$ & $\begin{array}{c}0,072 * * * \\
(0,028)\end{array}$ & $\begin{array}{c}0,106^{* * *} \\
(0,034)\end{array}$ \\
\hline Renda familiar*Masculino & - & $\begin{array}{c}0,005 \\
(0,003)\end{array}$ & & $\begin{array}{c}0,012 \\
(0,017)\end{array}$ \\
\hline Escolaridade da mãe & $\begin{array}{c}0,085^{* * *} \\
(0,002)\end{array}$ & $\begin{array}{c}0,071^{* * *} \\
(0,003)\end{array}$ & $\begin{array}{c}0,154 * * * \\
(0,032)\end{array}$ & $\begin{array}{c}0,182^{* * *} \\
(0,037)\end{array}$ \\
\hline Escolaridade da Mãe*Masculino & - & $\begin{array}{c}0,027 * * * \\
(0,004)\end{array}$ & - & $\begin{array}{c}0,032 \\
(0,017)\end{array}$ \\
\hline Escolaridade do Pai & $\begin{array}{c}0,051 * * * \\
(0,002)\end{array}$ & $\begin{array}{c}0,037 * * * \\
(0,002)\end{array}$ & $\begin{array}{c}0,029 * * * \\
(0,010)\end{array}$ & $\begin{array}{l}-0,002 \\
(0,014)\end{array}$ \\
\hline Escolaridade do pai* Masculino & - & $\begin{array}{c}0,026^{* * *} \\
(0,004)\end{array}$ & - & $\begin{array}{c}0,032^{* * *} \\
(0,009)\end{array}$ \\
\hline Tamanho da família & $\begin{array}{c}-0,196 * * * \\
(0,006)\end{array}$ & $\begin{array}{c}-0,196 * * * \\
(0,006)\end{array}$ & $\begin{array}{c}-0,379 * * * \\
(0,085)\end{array}$ & $\begin{array}{c}-0,501 * * * \\
(0,104)\end{array}$ \\
\hline Idade da mãe no nascimento & $\begin{array}{c}0,005^{* * *} \\
(0,002)\end{array}$ & $\begin{array}{c}0,005^{* * *} \\
(0,002)\end{array}$ & $\begin{array}{l}-0,011 \\
(0,008)\end{array}$ & $\begin{array}{c}-0,022^{* *} \\
(0,010)\end{array}$ \\
\hline Adolescente branco & $\begin{array}{c}0,144 * * * \\
(0,013)\end{array}$ & $\begin{array}{c}0,143^{* * * *} \\
(0,013)\end{array}$ & $\begin{array}{c}0,002 \\
(0,069)\end{array}$ & $\begin{array}{l}-0,093 \\
(0,086)\end{array}$ \\
\hline Masculino & $\begin{array}{c}-0,477 * * * \\
(0,012)\end{array}$ & $\begin{array}{c}-0,927 * * * \\
(0,115)\end{array}$ & $\begin{array}{c}-0,529 * * * \\
(0,030)\end{array}$ & $\begin{array}{c}-1,065^{* * *} \\
(0,307)\end{array}$ \\
\hline Trabalho do filho & $\begin{array}{c}0,306^{* * *} \\
(0,015)\end{array}$ & $\begin{array}{c}0,310^{* * *} \\
(0,015)\end{array}$ & $\begin{array}{c}0,564 * * * \\
(0,121)\end{array}$ & $\begin{array}{c}0,739^{* * *} \\
(0,149)\end{array}$ \\
\hline Constante & $\begin{array}{c}0,570^{* *} \\
(0,225)\end{array}$ & $\begin{array}{c}0,707^{* *} \\
(0,285)\end{array}$ & $\begin{array}{c}4,802^{* *} \\
(1.975)\end{array}$ & $\begin{array}{c}8,119^{* * *} \\
(2.52)\end{array}$ \\
\hline Efeito fixo de ano & Sim & $\operatorname{Sim}$ & $\operatorname{Sim}$ & $\operatorname{Sim}$ \\
\hline Efeito fixo de localidade & $\operatorname{Sim}$ & Sim & Sim & Sim \\
\hline Efeito fixo de coorte filho & Sim & Sim & Sim & Sim \\
\hline Efeito fixo de coorte mãe & Sim & Sim & $\operatorname{Sim}$ & Sim \\
\hline$R^{2}$ & 0.51 & 0,22 & 0,51 & 0,10 \\
\hline
\end{tabular}

Fonte: Resultados da pesquisa.

Nota: $\left({ }^{* *}\right),\left({ }^{* *}\right)$ e $\left({ }^{*}\right)$ indicam níveis de significância de $1 \%$, 5\% e 10\%, respectivamente. Erros padrões entre parênteses. 
Observa-se ainda que filhos de mães casadas têm em média 1,527 anos de estudo a menos que os filhos de outros arranjos, sendo esse resultado diferente do esperado, uma vez que em famílias monoparentais há uma redução do tempo dos pais, em cuidados de qualidade, com seus filhos. Esse efeito negativo também é encontrado por James-Burdumy (2005).

Além disso, os resultados aqui estimados sugerem que quanto maior o tamanho da família e quanto maior a idade da genitora no nascimento do filho, menor é o capital intelectual adquirido pelos adolescentes. Em relação à renda da família, nota-se que quanto maior o rendimento familiar, maior é a escolaridade dos filhos (com efeito marginal de 0,072), o que está de acordo com os resultados encontrados por Datcher-Loury (1988). $O$ fato de o adolescente trabalhar também afeta positivamente a sua educação. De acordo com Ravallion e Wodon (2000), o aumento na educação de meninos e de meninas está mais relacionado com a realocação do tempo entre lazer e estudo do que entre trabalho e educação.

Em relação ao diferencial educacional de sexo (especificações (2) na Tabela 2), a estimação via VI evidencia ausência de efeitos distintos e significativos das horas de trabalho da mãe sobre a escolaridade de filhos de sexos diferentes. Também não contribuem para introduzir diferenças educacionais entre os sexos o estado civil da mãe, os anos de estudo dela e a renda familiar. Contudo, verifica-se que a escolaridade do pai se relaciona de forma estatisticamente significativa ao diferencial educacional entre os sexos, sendo mais benéfico para os meninos do que para as meninas.

Mesmo que as horas trabalhadas pela mãe impactem negativamente no nível de escolaridade dos filhos, esse efeito pode ser reduzido devido ao aumento da renda da família, proveniente do emprego da mãe e pode influenciar de maneira distinta no gap educacional de gêneros. Esse mecanismo por meio do qual o trabalho materno pode ser benéfico à educação dos filhos é conhecido como canal da renda. Os resultados da especificação (2) via VI expressos na Tabela 2 mostram que o canal da renda não parece atingir de maneira diferente os filhos de sexos diferentes, o que corrobora o resultado encontrado por Fan, Fang e Markussen (2015).

Todavia, para explorar esse canal na educação dos filhos independente dos sexos, a Tabela 3 mostra os resultados de duas especificações adicionais: uma não controlando pela renda familiar (especificação (1)) e outra com a adição de tal controle (especificação (2)). Em relação à especificação (1), 
observa-se que o impacto referente ao aumento de uma hora trabalhada pela mãe foi significativo e negativo, com magnitude 0,093. Na especificação (2), esse efeito continua a ser significativo e negativo, embora apresente um ligeiro aumento $(0,095)$, o que indica que o aumento das horas trabalhadas tem um efeito positivo nos anos de estudo dos adolescentes pelo canal da renda no valor de 0,002. Contudo, esse efeito não é suficiente para reduzir totalmente o efeito negativo associado ao trabalho materno. A presença do canal da renda também é observada por Baum (2003).

Tabela 3 - Canal da renda nos anos de estudo dos adolescentes - 2004 a 2014

\begin{tabular}{lcc}
\hline & & VI \\
\cline { 2 - 3 } Horas de trabalho da mãe & $(1)$ & $(2)$ \\
& $-0,093^{* *}$ & $-0,095^{* * * *}$ \\
Renda familiar & $-0,042$ & $-0,044$ \\
Adolescente branco & - & $0,072^{* * *}$ \\
Dummies de background familiar & & $-0,028$ \\
Efeito fixo de ano & $\operatorname{Sim}$ & $\operatorname{Sim}$ \\
Efeito fixo de localidade & $\operatorname{Sim}$ & $\operatorname{Sim}$ \\
Efeito fixo de coorte filho & $\operatorname{Sim}$ & $\operatorname{Sim}$ \\
Efeito fixo de coorte mãe & $\operatorname{Sim}$ & $\operatorname{Sim}$ \\
\hline$R^{2}$ & $\operatorname{Sim}$ & $\operatorname{Sim}$ \\
\hline
\end{tabular}

Fonte: Resultados da pesquisa.

Nota: $\left({ }^{* *}\right),\left({ }^{* *}\right)$ e $\left({ }^{*}\right)$ indicam níveis de significância de $1 \%, 5 \%$ e $10 \%$, respectivamente.

Em suma, quanto maior a intensidade do trabalho materno, medido em horas de trabalho, menor o nível de capital humano do filho, medido em anos médios de estudo com base na última série concluída com aprovação (esse efeito é reduzido, mas não eliminado pelo canal da renda). Todavia, esse resultado não é diferente para os filhos de sexos diferentes, não contribuindo assim para a reversão do gap educacional de gênero que tem ocorrido no Brasil nos últimos anos. 


\section{Conclusões}

Neste estudo, foram avaliadas duas importantes questões presentes nos recentes debates acerca do desenvolvimento econômico: a inserção das mães no mercado de trabalho e os resultados em termos de escolaridade dos seus filhos. Objetivou-se verificar se existem relações entre a participação da mãe no mercado de trabalho e a educação de seus filhos adolescentes; ainda, se tal efeito se manifesta de maneira diferente entre filhos e filhas.

Na literatura nacional, há poucas evidências sobre o tema analisado neste artigo, já o oposto ocorre na literatura internacional. Os resultados encontrados no âmbito internacional são vastos, mas díspares. Uma das possíveis explicações para a variedade desses resultados deve-se à endogeneidade presente na decisão de trabalho das mães (e a ausência de controle do problema). Essa decisão é, possivelmente, afetada por fatores observáveis e não observáveis (como cansaço, estresse, ausência de divisão igualitária de tarefas domésticas, habilidade, empreendedorismo e iniciativa das mães) que podem se relacionar ao nível de escolaridade dos filhos. Com uma estratégia de identificação que buscou por variações exógenas nas horas trabalhadas pelas mães das regiões metropolitanas brasileiras (por meio das condições locais do mercado de trabalho), neste trabalho, evidenciou-se que a falha em levar tal endogeneidade em consideração resulta em estimativas que negligenciam o verdadeiro efeito do trabalho materno.

Os resultados encontrados sugerem que uma maior intensidade do trabalho materno associa-se negativamente ao nível de escolaridade dos filhos. Além disso, não parece haver efeitos distintos entre filhos de sexos diferentes. Diversos fatores podem explicar esse resultado, como o possível cansaço da mãe, associado a maiores quantidades de horas de trabalho, e a predominância de jornadas duplas entre as mulheres (que muitas vezes não contam com divisão igualitária de tarefas para o cuidado da casa e das crianças entre seus cônjuges).

Esses resultados apontam para a importância de fornecer às mulheres condições para que exerçam plenamente suas profissões, sem que necessariamente seus filhos tenham resultados inferiores em termos educacionais. Desenvolver meios para que elas tenham a opção de seguir suas carreiras e crescerem profissionalmente, ao mesmo tempo em que seus filhos sejam bem desenvolvidos na carreira educacional é de suma importância. 
Em termos metodológicos, devem-se reconhecer algumas limitações deste estudo. Variáveis adicionais poderiam ter sido incluídas na equação que explica a escolaridade dos adolescentes, a fim de isolar melhor o efeito de interesse. Essas seriam: o tempo de qualidade que os pais dedicam aos filhos, a ajuda e incentivo à educação, a presença de outras pessoas que exercem esse cuidado, como irmãos, professores e avós, entre outras. No entanto, reitera-se que não foi possível estender a análise nesse sentido devido aos dados disponíveis.

\section{Referências}

Aguas, Marina Ferreira Fortes. 2009. “A importância da composição dos filhos para a participação da mulher na força de trabalho - uma análise para o Brasil de 1992 a 2008”. (MSc diss., UFF 2009).

Anazawa, Leandro, Guedes, Marcelo Sanchez, Komatsu, Bruno Kawaoka, and Menezes-Filho, Naercio Aquino. 2016. "A Loteria da Vida: Examinando a Relação entre a Educação da Mãe e a Escolaridade do Jovem com Dados Longitudinais do Brasil’. Insper - Centro de Políticas Públicas, Policy Paper 22.

Anderson, Patricia M., Kristin F. Butcher, and Phillip B. Levine. 2003. "Maternal employment and overweight children." Journal of health economics 22(3): 477-504.

Aquino, Juliana Maria, and Elaine Toldo Pazello. 2011. "Trabalho materno e desempenho educacional das crianças: uma análise da probabilidade de aprovação escolar.” Pesquisa e Planejamento Econômico 41(1): 71-100.

Arouck, Jean Henrique Santana S. 2015. "Trabalho materno e desempenho educacional: uma análise para o ensino fundamental gaúcho”. (MSc diss., Pontifícia Universidade Católica do Rio Grande do Sul 2015).

Baum II, Charles L. 2003. "Does early maternal employment harm child development? An analysis of the potential benefits of leave taking." Journal of labor Economics 21(2): 409-448.

Baum, Charles L. 2004. "The long-term effects of early and recent maternal employment on a child's academic achievement." Journal of Family Issues 25(1): 29-60.

Baydar, Nazli, and Jeanne Brooks-Gunn. 1991. "Effects of maternal employment and child-care arrangements on preschoolers' cognitive and behavioral outcomes: Evidence from the Children of the National Longitudinal Survey of Youth.” Developmental psychology 27(6): 932-945.

Beltrão, Kaizô Iwakami, and José Eustáquio Diniz Alves. 2009. "A reversão do hiato de gênero na educação brasileira no século XX.” Cadernos de Pesquisa 39(136): 125-156.

Blau, Francine D., and Adam J. Grossberg. 1990. "Maternal labor supply and children's cognitive development”. National Bureau of Economic Research 1990.

Brooks-Gunn, Jeanne, Wen-Jui Han, and Jane Waldfogel. 2002. "Maternal employment and child cognitive outcomes in the first three years of life: The NICHD study of early child care." Child development 73(4): 1052-1072.

Cameron, A. Colin, and Pravin K. Trivedi. 2005. "Microeconometrics: methods and applications". Cambridge University Press 2005.

Cardoso, Fernando Luiz, Samantha Sabbag, and Thais Silva Beltrame. 2007. "Prevalência de transtorno de déficit de atenção/hiperatividade em relação ao gênero de escolares." Revista Brasileira de Cineantropometria e Desempenho Humano 9(1): 52-59.

Datcher-Loury, Linda. 1988. "Effects of mother's home time on children's schooling." The review of economics and statistics 70(3): 367-373. 
Duflo, Esther. 2012. “Women empowerment and economic development.” Journal of Economic Literature 50(4): 1051-1079.

Fan, Xiaodong, Hanming Fang, and Simen Markussen. 2015. “Mothers' Employment and Children's Educational Gender Gap". National Bureau of Economic Research 2015.

González de San Román, A.; De La Rica Goiricelaya S. 2001. “Gender Gaps in Han, Wen-Jui, Jane Waldfogel, and Jeanne Brooks-Gunn. The effects of early maternal employment on later cognitive and behavioral outcomes." Journal of Marriage and Family 63(2): 336-354.

Hill, M. Anne, and June O'Neill. 1994. "Family endowments and the achievement of young children with special reference to the underclass." Journal of Human Resources 29(4): 1064-1100.

James-Burdumy, Susanne. 2005. "The effect of maternal labor force participation on child development.” Journal of Labor Economics 23(1): 177-211.

Leone, Eugene Troncoso. 2000. "Renda familiar e trabalho da mulher na Região Metropolitana de São Paulo nos anos 80 e 90". In Trabalho e gênero: mudanças, permanências e desafios, 384. São Paulo: Editora.

Muller, Chandra. 1998. "Gender differences in parental involvement and adolescents' mathematics achievement." Sociology of Education 71(4): 336-356.

"PISA Test Scores: The Impact of Social Norms and the Mother's Transmission of Role Attitudes. IZA Discussion Paper 6338, 2012.

Ravallion, Martin, and Quentin Wodon. 2000. "Does child labour displace schooling? Evidence on behavioral responses to an enrollment subsidy." The economic journal 110(462): 158-175.

Ruhm, Christopher J. 2008. "Maternal employment and adolescent development." Labour Economics 15(5): 958-983.

Souza, Laetícia Rodrigues, and Rios-Neto, Eduardo Luiz Gonçalves. 2008. "O efeito de filhos sobre a oferta de trabalho dos pais sob uma perspectiva de gênero." Anais do XVI Encontro Nacional de Estudos Populacionais, Caxambu, MG.

UNESCO. 2015. "Relatório de Monitoramento Global”. Brasília: UNESCO, CONSED, Ação Educativa.

Vieira, Caterina Soto, Cabanas, Pedro, Menezes-Filho, Naercio, and Komatsu, Bruno Kawaoka. 2016. "Como as Mudanças no trabalho e Renda dos Pais afetam as Escolhas entre Estudo e Trabalho dos Jovens?" Pesquisa e Planejamento Econômico 46(3): 33-61.

Wooldridge, Jeffrey. M. 2002. "Econometric Analysis of Cross Section and Panel Data." Cambridge, MA: MIT Press. 